\section{Nova validação semântico-cultural e estudo psicométrico da CASP-19 em adultos e idosos brasileiros}

\author{
New semantic-cultural validation and \\ psychometric study of the CASP-19 scale in adult \\ and elderly Brazilians
}

Nueva validación semántico-cultural y estudio
psicométrico de la CASP-19 en adultos y ancianos
brasileños

\section{Resumo}

Os objetivos foram investigar indicadores psicométricos de validade de nova versão da CASP-19 para brasileiros com 55 anos e mais e estudar relações entre pontuações na escala e sexo, idade, escolaridade e status conjugal. Trezentos e sessenta e oito frequentadores de programas educacionais para a $3 a$ idade responderam à CASP-19 (controle, autonomia, autorrealização e prazer), traduzida e adaptada do inglês por cinco especialistas e testada em 19 mulheres $(\alpha=0,730)$. Os dados foram submetidos a análises fatoriais exploratórias (AFE) e confirmatórias (AFC) pelo método de equações estruturais para variáveis latentes; de consistência interna e de correlação com instrumentos de conteúdo similar. Foram comparadas as pontuações dos grupos de sexo, idade, escolaridade e status conjugal. AFC produziram modelo com 19 itens e 2 fatores (autorrealização/prazer e controle/autonomia), com bons indices de ajustamento $(G F I=0,8 ; A G F I=0,7606 ; C F I=0,7241 ; N N F I=0,6876$; $S R M R=0.0902 ; R M S E A=0,0928 ;$ IC90\%: 0,0827-0,1031). Os $\alpha$ de Cronbach foram 0,837 para o fator 1 e 0,670 para o 2; 0,874 na escala total para os de 9 anos ou mais de escolaridade, 0,834 para os de 5 a 8 anos $e$ 0,772 para os de 1 a 4 anos. Foram observadas correlações altas e significativas com os escores em escalas de satisfação e felicidade subjetiva. Homens tiveram pontuação mais alta em autorrealização/prazer do que mulheres; os mais velhos $e$ os mais escolarizados, pontuação mais baixa em controle/autonomia; os sem cônjuge, mais alta em controle/autonomia. A nova versão da CASP-19 foi eficaz para avaliar a qualidade de vida percebida em indivíduos de 55 anos e mais, residentes nas regiões Sudeste, Sul e Nordeste do país.

Qualidade de Vida; Reprodutibilidade dos Testes; Envelhecimento
QUESTÕES METODOLÓGICAS

METHODOLOGICAL ISSUES

Anita Liberalesso Neri 1

Flávia Silva Arbex Borim 1

Samila Sathler Tavares Batistoni 1,2

Meire Cachioni 1,2

Dóris Firmino Rabelo 3

Arlete Portella Fontes 1

Mônica Sanches Yassuda 2

doi: 10.1590/0102-311X00181417

Correspondência

F. S. A. Borim

Programa de Pós-graduação em Gerontologia, Faculdade de Ciências Médicas, Universidade Estadual de Campinas.

Av. Tessalia Vieira de Camargo 126, Campinas, SP 13083-887, Brasil.

flarbex@hotmail.com

${ }^{1}$ Faculdade de Ciências Médicas, Universidade Estadual de Campinas, Campinas, Brasil.

2 Universidade de São Paulo, São Paulo, Brasil.

3 Universidade Federal do Sudoeste da Bahia, Vitória da

Conquista, Brasil. 


\section{Introdução}

CASP-19 é o acrônimo que designa uma escala com 19 itens destinada à avaliação de qualidade de vida percebida em pessoas com 55 anos ou mais. Ela é referenciada aos construtos psicológicos: controle, definido como a capacidade de intervir ativamente no ambiente; autonomia, como o direito de um indivíduo de ser livre de interferências indesejáveis por parte de outrem; e autorrealização e prazer, como manifestações de agência e atividade, fundamentais à livre participação do indivíduo na vida social 1. Adota como fundamento o ponto de vista ontológico assumido por Maslow 2, segundo o qual o fato de serem humanas determina que as pessoas compartilhem um conjunto de necessidades básicas que desempenham um papel orientador em relação à sua personalidade e ao seu desenvolvimento. Diferentemente do modelo de Maslow 2, o da CASP-19 não é hierárquico.

A construção e os primeiros estudos de validação da CASP-19 foram realizados com amostras de três estudos ingleses prospectivos: sobreviventes da coorte do estudo de Boyd-Orr ${ }^{3}$, participantes da 1a onda de medidas do English Longitudinal Study of Aging (ELSA) 4 e participantes da 11a onda de medidas do British Household Panel Survey ${ }^{5}$. Na primeira amostra, foram aplicados a primeira versão da escala, com 22 itens, e o Life Satisfaction Index ${ }^{6}$. Foram observados correlação significativa entre as duas escalas $(0,60$ a 0,70) e índices de 0,60 a 0,80 nos quatro domínios da CASP. Com base em análise fatorial de segunda ordem, a escala foi reduzida a 19 itens com cargas fatoriais altas $(0,71$ a 0,78$)$ e a um único fator latente, representativo de qualidade de vida. Os outros dois estudos ofereceram contexto para a realização de análises fatoriais confirmatórias (AFC) cuja melhor solução foi uma versão de 12 itens estruturados em quatro fatores 7 . Hamrem et al. 8 encontraram índices aceitáveis de consistência interna para controle $(\alpha=0,67)$, autonomia $(\alpha=0,76)$, autorrealização $(\alpha=0,70)$ e prazer $(\alpha=0,63)$, dispostos em 11 itens. Em estudo com amostras do Health, Alcohol and Psychosocial Factors in Eastern Europe (HAPIEE) 9, a estrutura de 19 itens e 4 fatores não teve bom ajustamento. No estudo de Oluboyede \& Smith 10, a análise de Rasch revelou que quatro itens não se adequavam ao modelo de 19 itens, e que o modelo de quatro fatores não se ajustava aos dados. Novas AFC resultaram em estrutura de dois fatores reunindo os itens de controle e autonomia no fator 1 e de autorrealização e prazer no fator $211,12,13,14,15$. Ou seja, os dados de estudos psicométricos sobre as variáveis latentes à CASP-19 não são consensuais. A escala continua gerando estudos metodológicos.

A CASP-19 tem sido usada como medida dependente em estudos realizados em vários países de língua inglesa, entre eles, a Inglaterra 4,12, os Estados Unidos 16, a Irlanda 11,13,15 e a Nova Zelândia 17, e em estudos longitudinais e transnacionais europeus, tais como o Health, Aging and Retirement in Europe SHARE 18 e o HAPIEE 9. Em artigo de revisão que marca os dez anos de criação da escala, Hyde et al. 19 mencionam que ela tem sido usada em mais de vinte países localizados nos cinco continentes e tem produzido numerosos estudos de validação.

Lima et al. 20 realizaram a primeira adaptação semântico-cultural da CASP-19 para a língua portuguesa falada no Brasil. Aplicaram à versão resultante a amostra de 87 idosos ( $\mu=75,6 \pm 0,7$ anos) residentes em Recife, Pernambuco. A consistência interna foi baixa, mesmo com remoção de itens. Análises fatoriais produziram uma estrutura de 16 itens e quatro fatores. O fator prazer contribuiu menos para explicar a variabilidade dos itens do que na versão inglesa, dados que os autores creditam a diferenças culturais. Essa versão foi incluída no protocolo do EpiFloripa Idoso, Santa Catarina, Brasil 21,22,23. O Estudo Longitudinal de Saúde do Idoso (ELSI-Brasil) 24 adotou tradução própria da CASP- 19.

Considerando que o Brasil é país de dimensões continentais, com habitantes de várias etnias e subculturas, refletidas em numerosas variações linguísticas da língua portuguesa, julgamos oportuno realizar nova validação semântico-cultural, com amostra maior e mais diversificada quanto à origem cultural e geográfica, bem como novas análises psicométricas da nova versão do instrumento. Acreditamos que o novo estudo poderá enriquecer o conhecimento disponível sobre a generalidade intercultural dos elementos do construto qualidade de vida subjacentes à CASP-19 e sobre sua apresentação em cultura diversa daquela na qual a escala foi construída e inicialmente validada.

Este estudo tem como objetivos: (1) descrever procedimentos e resultados de nova adaptação semântico-cultural da escala CASP-19 para brasileiros de 55 anos e mais e da avaliação inicial de sua validade interna; (2) investigar evidências de validade de construto e de validade interna do instrumento; (3) investigar relações entre os valores da distribuição dos dados da CASP-19 com os valores produzidos por instrumentos de conteúdo similar, em busca de indicadores de validade convergente; 
e (4) caracterizar o perfil de qualidade de vida percebida na amostra de adultos e idosos, considerando sexo, idade, escolaridade e status conjugal.

\section{Métodos}

$\mathrm{O}$ estudo compreendeu quatro fases. Na primeira, participaram dois grupos de colaboradores. $\mathrm{O}$ primeiro foi composto por dois psicólogos brasileiros bilíngues (T1 e T2), um informado e o outro não informado sobre a teoria subjacente à CASP-19; um psicólogo brasileiro fluente em inglês e informado sobre a CASP-19 (T3), e dois tradutores profissionais bilíngues não informados (T4 e T5). O segundo contou com 19 mulheres de um curso de formação de cuidadores de idosos, 17 com 15 ou mais anos de escolaridade e 2 com 11; dez com 44-59 anos e 9 com 60-74 anos ( $\mu=59,4 \pm 5,7)$.

Para o cumprimento dos objetivos das três fases subsequentes, foi composta amostra de conveniência com 368 indivíduos com 55 anos e mais que frequentavam Universidades da Terceira Idade ou cursos de educação em saúde, de cidades das regiões Sudeste (54,3\%), Nordeste, (36,7\%) e Sul (9\%). Foram adotados como critérios de exclusão: analfabetismo, incapacidade para ler e responder por escrito e dificuldade para compreender as instruções, relatadas pelos participantes potenciais, pelos professores e coordenadores ou por membros da equipe de pesquisa. Entre os participantes, $86 \%$ eram mulheres; $22 \%$ tinham de 44-59 anos; 48\%, de 60-69 anos; 24,7\%, de 70-79 anos; e 5,3\% tinham 80 anos e mais ( $\mu=65,6 \pm 8,0)$; $22 \%$ tinham de $1-4$ anos; $11,5 \%$, de 5-8 anos; $24,4 \%$, de $9-11$ anos; e $42,4 \%$, de 12 ou mais anos de escolaridade; 47,2\% viviam com cônjuge ou companheiro(a); 14,6\% eram solteiros; 25\%, viúvos; e 13,1\%, divorciados.

A aplicação dos instrumentos foi coletiva, de lápis e papel, nas salas de atividades dos programas. Todos os participantes assinaram um termo de consentimento livre e esclarecido. O projeto de pesquisa foi aprovado pelo Comitê de Ética em Pesquisa da Universidade Estadual de Campinas, em 7 de outubro de 2015, e registrado na Plataforma Brasil sob o C.A.A.E. 35471714.1.0000.5404.

\section{1ạ fase: validação semântico-cultural e estudo preliminar da consistência interna da CASP-19}

Os processos de tradução, retrotradução e adequação semântico-cultural foram realizados de acordo com normas correntes 25 . Inicialmente, T2 e T4 trabalharam independentemente e, depois, em conjunto na tradução da CASP-19 do inglês para o português. A versão de consenso resultante foi submetida à retrotradução por $\mathrm{T} 1 \mathrm{e} \mathrm{T} 5$, atuando separadamente. As duas retrotraduções foram cotejadas por T3; e os resultados, discutidos com T1 e T5. A tarefa foi dada como completa quando T1, T3 e T5 concordaram com a redação de todos os itens, tendo em vista sua adequação semântico-cultural.

A versão consolidada da tradução foi aplicada ao grupo de 19 mulheres, em formato de 19 escalas de 4 pontos cada uma (de $0=$ nunca a 3 = sempre). Trata-se de medida somativa que pode variar de 0 a 57. As pontuações mais altas são consideradas indicadoras de alta qualidade de vida percebida. Seis itens $(1,2,4,6$, 8, e 9) são redigidos no negativo e invertidos na análise de dados; 13 são positivos $1,7$. Foi calculada a confiabilidade interna, mediante análise das correlações múltiplas entre os itens, adotando-se, como indicador, o $\alpha$ de Cronbach.

\section{2a fase: investigação de indicadores de validade de construto}

Foram realizadas análises fatoriais exploratórias (AFE) e AFC, com o duplo propósito de testar a estrutura da CASP-19 e de obter a solução fatorial com melhor ajuste 26 . Por sorteio, os 368 participantes foram divididos em duas subamostras equivalentes, considerando-se as variáveis cidade de origem, sexo, idade, escolaridade e status conjugal. As AFE foram feitas sobre os dados da primeira subamostra $(\mathrm{n}=150)$, e as AFC, sobre os dados da segunda $(\mathrm{n}=218)$. Em ambas as circunstâncias, foi analisada a consistência interna dos fatores.

As AFC adotaram o modelo de equações estruturais para variáveis latentes, baseado em parâmetros fixos (cargas fatoriais iguais a zero) e parâmetros livres a serem estimados (cargas fatoriais diferentes de zero) 26. Os indicadores de adequação do ajuste do modelo aos dados da amostra foram obtidos por meio dos testes qui-quadrado e razão de qui-quadrado ( $\left.\chi^{2} / \mathrm{GL}\right)$ e indicados pelo 
índice de qualidade do ajuste (GFI - goodness of fit index), pelo índice ajustado de qualidade do ajuste (AGFI - adjusted for degrees of freedom), pela raiz do resíduo quadrático médio (SRMR - standardized root mean square residual), pela raiz do erro quadrático médio de aproximação (RMSEA - root mean square error of approximation), pelo índice de ajuste comparativo de Bentler's (CFI - Bentler's comparative fit index) e pelo índice de ajuste não ponderado de Bentler e Bonett (NNFI - Bentler er Bonett's non-normed fit index) 26. Para analisar a qualidade do ajuste aos fatores propostos, foram feitos testes de significância para as cargas fatoriais, com valor de t estabelecido como $>1.96$ ). Para propor modificações nos fatores por meio da exclusão ou da realocação de itens, foram utilizados os testes de Wald e do multiplicador de Lagrange.

\section{3a fase: busca de indicadores de validade convergente}

Foram aplicadas duas medidas de bem-estar subjetivo: a Escala de Satisfação com a Vida 27 e a Escala de Felicidade Subjetiva 28, ambas de natureza unidimensional. A primeira avalia os componentes cognitivo e de julgamento do bem-estar subjetivo. Contém 5 itens tipo Likert, com 7 pontos cada um (de $1=$ discordo fortemente a 7 = concordo fortemente). A segunda avalia a felicidade do ponto de vista dos respondentes. Contém quatro escalas com sete pontos e três âncoras conceituais cada uma (muito $\mathrm{x}$ nem muito e nem pouco $\mathrm{x}$ pouco). Foram comparadas as pontuações totais dos 368 participantes nas três escalas e realizadas análises de correlação entre elas.

\section{4a fase: relações entre qualidade de vida percebida, indicada pela CASP-19, e as variáveis sexo, idade, escolaridade e status conjugal}

Foram estatisticamente comparadas as pontuações obtidas na CASP-19 pelos homens e pelas mulheres, pelos participantes que tinham idade de 44-59 anos, de 60-74 anos e de 75 anos ou mais, pelos que tinham de 1-8 anos, ou 9 ou mais anos de escolaridade, e pelos que viviam com cônjuge ou companheiro(a), em comparação com os solteiros, viúvos e divorciados. Para tanto, foram utilizados os testes de Mann-Whitney, Kruskal-Wallis e post hoc de Dunn.

As análises estatísticas foram realizadas por meio do SAS (https://www.sas.com), versão 9.2, e do IBM SPSS, versão 22 (https://www.ibm.com).

\section{Resultados}

\section{Fase 1}

Durante o processo de tradução e adaptação da CASP-19, foram observadas três discordâncias entre os itens originais e a primeira retrotradução e três discrepâncias entre o original em inglês e a segunda retrotradução. Os tradutores usaram verbos ou construções diferentes, mas com o mesmo sentido e discutiram possibilidades até chegar a um consenso. Na versão final em português do Brasil, o item 10 foi alterado de "Eu anseio por viver cada dia" para "Eu tenho vontade de viver cada dia"; o 15, de "Eu me sinto cheio de energia ultimamente" por "Ultimamente, eu tenho me sentido cheio de energia", o 14 "Quando eu faço um balanço da minha vida..." por "Quando avalio minha vida até aqui..." e o 18 de "Eu tenho esperança no futuro" por "Eu acho que o futuro reserva coisas boas para mim". Foram feitos ajustes linguísticos nos itens 2,3 e 4 e 7 , substituindo-se a forma coloquial "eu me sinto" pela forma "sinto-me".

O grupo de mulheres sugeriu traduzir os advérbios often, sometimes, not often e never como sempre, quase sempre, às vezes e nunca para tornar mais clara a diferença entre as frequências, sugestão acatada por esta pesquisa. Sugeriram que todos os itens tivessem a mesma direção para facilitar a resposta, mas, neste estudo, foi mantida a redação original, tanto para os seis itens negativos (1, 2, 4, 6, 8 e 9), que foram invertidos na análise de dados, como para os 13 positivos. 


\section{Fase 2}

Compreendeu a realização de AFE sobre as respostas à CASP-19 observadas na primeira metade da amostra ( $\mathrm{n}=150)$. Inicialmente, foi calculado o índice MSA (measure of sampling adequacy) de KaiserMeyer-Olkin (KMO) para esses dados, o qual resultou em um valor de 0,840, indicativo de boa consistência. A matriz inicial de correlações produzida pela análise foi submetida à rotação Varimax. Pelo critério de seleção de fatores com autovalor maior que 1, foram obtidos cinco fatores, que explicaram $61.2 \%$ da variabilidade dos dados. Com base no teste de resíduos, optou-se por fixar a extração em três fatores, que explicaram $49,6 \%$ da variabilidade total dos itens.

A carga mínima aceita para manutenção no modelo foi 0,30. Os itens 15 ("Ultimamente, eu tenho me sentido cheio de energia"), 7 ("Sinto-me realizado com o que faço") e 16 ("Eu escolho fazer coisas que nunca fiz antes") obtiveram carga > 0,30 em mais de um fator, e, com base em critério teórico, foram alocados respectivamente nos fatores 1, 3 e 2. O fator 1 agregou itens originalmente alocados nos fatores prazer e autonomia, e o fator 2, os de autonomia e controle com conotação negativa. No fator 3, ficaram dois itens do fator original autonomia, dois de controle e um de autorrealização. $\mathrm{O}$ item 11 ("Eu sinto que minha vida tem sentido") foi o de maior comunalidade, isto é, teve 72,2\% de sua variabilidade explicada pelos fatores, e o item 4 ("Sinto-me deixado de lado"), com 20,1\%, foi o de menor comunalidade.

A composição do fator 3 pareceu complementar aos significados subjacentes aos fatores 1 e 2, sugerindo a extração de dois fatores. A estrutura fatorial resultante redistribuiu os itens do fator 3 pelos fatores 1 e 2 . Os itens 15 e 16 obtiveram carga < 0,30 em mais de um fator e foram alocados no 1, que tem a maior carga. O item 11 foi, novamente, o de maior comunalidade, isto é, teve 65,8\% de sua variabilidade explicada pelos fatores; o 4 foi o de menor comunalidade (17,9\%). A solução em dois fatores explicou 42,3\% da variabilidade total dos dados da escala (Tabela 1).

Foi feita AFC com os dados da segunda subamostra ( $n=218)$. O índice de ajuste (SRMR) desses dados alcançou 0,083, considerado como adequado. Primeiro, foi testada a solução em três fatores obtida na fase anterior. Todos os itens apresentaram saturação suficiente para manutenção nos respectivos fatores, com exceção do 16, que foi transferido do fator 3 para o 1. Depois de calculados os índices de ajuste, o modelo foi novamente rotacionado. Embora os resultados tenham se mantido, pareceu teoricamente interessante retornar o item 15 para o fator 2. Depois de nova rotação, todos os valores do teste de adequação do ajuste para a AFC foram estatisticamente significantes para $\mathrm{p}<$ 0,001, mas as estatísticas de ajuste tiveram uma discreta piora, o que determinou o retorno do item 15 para o fator 1, encerrando-se a fase de teste do modelo de três fatores.

Em seguida, foi testada a composição da CASP-19 com dois fatores, cujo valor inicial de ajuste (SRMR) foi de 0,085. Todos os itens apresentaram saturação suficiente para manutenção nos respectivos fatores, com exceção do 16, cuja mudança do fator 3 para o fator 1 foi testada em nova análise fatorial. De modo geral, os resultados anteriores se mantiveram. Depois de novas manipulações, o item 15 foi realocado de volta no fator 1. Prevaleceu a solução fatorial apresentada na Tabela 2. Os itens dos domínios autorrealização e prazer predominaram no fator 1, e os dos domínios controle e autonomia, no fator 2 .

\section{Fase 3}

Os participantes pontuaram alto na CASP-19, nas escalas de satisfação com a vida e de felicidade subjetiva. Na CASP-19, para os escores totais da amostra, que variaram entre 18 e 57, a mediana foi de 48,0, e a média, de 46,3 7,4. Na Escala de Felicidade Subjetiva, para variação entre 7 e 28 pontos, a mediana foi de 21,9, e a média, de 21,9 4,2. Na Escala de Satisfação com a Vida, a mediana foi de 25,9, e

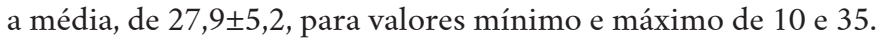

Os valores das correlações foram de 0,453 ( $\mathrm{p}<0,001)$ para a comparação entre a CASP-19 e a escala de felicidade, de 0,475 ( $\mathrm{p}<0,001)$ para a comparação entre a CASP-19 e a escala de satisfação. As análises de correlação bivariada entre os itens da CASP-19 e os das escalas de felicidade e de satisfação resultaram em valores estatisticamente significantes $(\mathrm{p}<0,05)$ para a grande maioria deles. Ausência de correlações estatisticamente significantes foram observadas entre o item 2 da Escala de 
Tabela 1

Estruturas resultantes das análises fatoriais exploratórias aplicadas aos dados da 1a subamostra (n = 150). CASP-19, Brasil, 2018.

\begin{tabular}{|c|c|c|c|}
\hline Cargas & Fator 1 & Cargas & Fator 1 \\
\hline 0,835 & 11. Eu sinto que minha vida tem sentido. & 0,800 & 11. Eu sinto que minha vida tem sentido. \\
\hline 0,781 & 10. Eu tenho vontade de viver cada dia. & 0,784 & 12. Eu gosto das coisas que faço. \\
\hline 0,741 & 12. Eu gosto das coisas que faço. & 0,743 & 10. Eu tenho vontade de viver cada dia. \\
\hline 0,714 & 14. Quando avalio a minha vida até aqui, eu me sinto feliz. & 0,731 & 7. Sinto-me realizado com o que faço. \\
\hline 0,674 & 17. Sinto-me satisfeito com as minhas realizações. & 0,691 & 17. Sinto-me satisfeito com as minhas realizações. \\
\hline 0,660 & 13. Eu gosto de estar na companhia de outras pessoas. & 0,689 & 14. Quando avalio a minha vida até aqui, eu me sinto feliz. \\
\hline 0,656 & 19. Eu acho que o futuro reserva coisas boas para mim. & 0,676 & 15. Ultimamente, eu tenho me sentido cheio de energia. \\
\hline 0,647 & $\begin{array}{l}\text { 18. Eu sinto que a vida me proporciona muitas } \\
\text { oportunidades. }\end{array}$ & 0,662 & $\begin{array}{c}\text { 18. Sinto que a vida me proporciona muitas } \\
\text { oportunidades. }\end{array}$ \\
\hline \multirow[t]{5}{*}{0,552} & 15. Ultimamente, eu tenho me sentido cheio de energia. & 0,600 & 19. Eu acho que o futuro reserva coisas boas para mim. \\
\hline & & 0,562 & 13. Eu gosto de estar na companhia de outras pessoas. \\
\hline & & 0,514 & 5. Eu posso fazer as coisas que quero. \\
\hline & & 0,412 & 16. Eu escolho fazer coisas que nunca fiz antes. \\
\hline & & 0,389 & 3. Sinto-me livre para planejar o futuro. \\
\hline
\end{tabular}

Fator 2

\begin{tabular}{|c|c|}
\hline 0,699 & 8. Minha saúde me impede de fazer as coisas que quero. \\
\hline 615 & $\begin{array}{l}\text { 9. A falta de dinheiro me impede de fazer as coisas que } \\
\text { quero. }\end{array}$ \\
\hline 0,589 & $\begin{array}{l}\text { 6. As responsabilidades familiares me impedem de fazer o } \\
\text { que quero. }\end{array}$ \\
\hline 0,556 & $\begin{array}{l}\text { 2. Sinto que não tenho controle sobre o que acontece } \\
\text { comigo. }\end{array}$ \\
\hline 530 & 1. Minha idade me impede de fazer as coisas que gostaria. \\
\hline & 4. Sinto-me deixado de lado. \\
\hline
\end{tabular}

Fator 2

8. Minha saúde me impede de fazer as coisas que quero.

9. A falta de dinheiro me impede de fazer as coisas que quero.

6. As responsabilidades familiares me impedem de fazer o que quero.

2. Sinto que não tenho controle sobre o que acontece comigo.

1. Minha idade me impede de fazer as coisas que gostaria. 4. Sinto-me deixado de lado.

Fator 3

\begin{tabular}{ll}
\hline 0,827 & 5. Eu consigo fazer as coisas que quero. \\
0,688 & 3. Sinto-me livre para planejar o futuro. \\
0,592 & 7. Sinto-me realizado com o que faço. \\
0,398 & 16. Eu escolho fazer coisas que nunca fiz antes. \\
\hline
\end{tabular}

Nota: medidas de adequação do ajuste obtidas: qui-quadrado = 433,37; qui-quadrado DF = 151; valor de $\mathrm{p}<0,000 ;$ qui-quadrado ratio = 2,87; Goodness of Fit Index (GFI) =0,8098; GFI Adjusted for Degrees of Freedom (AGFI) =0,7606; Bentler's Comparative Fit Index (CFI) = 0,7241; Bentler \& Bonett's 26 $(\mathrm{NNFI})=0,6876$; Standardized Root Mean Square Residual $(\mathrm{SRMR})=0,0902 ; \mathrm{RMSEA}$ estimate $=0,0928 / \mathrm{IC} 90 \%:(0,0827-0,1031)$.

Medidas de adequação do ajuste esperadas: valor de $p>0,05 ;$ razão qui-quadrado $<2,0 ; \mathrm{GFI} \geq 0,85 ; \mathrm{AGFI} \geq 0,80 ; \mathrm{CFI} \geq 0,90 ; \mathrm{NNFI} \geq 0,90 ; \mathrm{SRMR} \leq 0,10$; RMSEA $\leq 0,08$ 26;

Itens x domínio na CASP-19 original: controle: 1 a 4; autonomia: 5 a 9; prazer: 10 a 14 e autorrealização: 15 a 19.

Felicidade Subjetiva (que envolvia comparação social) e os itens 1 ("Minha idade me impede de fazer as coisas que gostaria"), 5 ("Eu consigo fazer as coisas que quero"), 6 ("As responsabilidades familiares me impedem de fazer o que quero") e 9 ("A falta de dinheiro me impede de fazer as coisas que quero") da CASP-19; entre o item 4 da escala de felicidade (em que medida o respondente acredita que não é muito feliz) e os itens da CASP-19 de números 1 ("Minha idade me impede de fazer as coisas que gostaria”), 2 ("Sinto que não tenho controle sobre o que acontece comigo"), 3 ("Sinto-me livre para planejar o futuro"), 4 (“Sinto-me deixado de lado”), 6 (“As responsabilidades familiares me impedem de fazer o que quero"), 10 (“Eu tenho vontade de viver cada dia”), 13 ("Eu gosto de estar na companhia de outras pessoas") e 15 (“Ultimamente, eu tenho me sentido cheio de energia”). Observou-se 
Tabela 2

Estrutura resultante da análise fatorial confirmatória aplicada aos dados da 2a subamostra $(n=218)$. CASP-19, Brasil, 2016.

\begin{tabular}{|c|c|c|c|}
\hline Fator & Carga & $\mathbf{t} *$ & Item \\
\hline \multirow[t]{13}{*}{1} & 0,682 & 10,71 & 11. Eu sinto que minha vida tem sentido. \\
\hline & 0,666 & 10,37 & 12. Eu gosto das coisas que faço. \\
\hline & 0,651 & 10,08 & 15. Ultimamente, eu tenho me sentido cheio de energia. \\
\hline & 0,605 & 9,20 & 18. Eu sinto que a vida me proporciona muitas oportunidades. \\
\hline & 0,588 & 8,87 & 14. Quando avalio a minha vida até aqui, eu me sinto feliz. \\
\hline & 0,560 & 8,37 & 19. Eu acho que o futuro reserva coisas boas para mim \\
\hline & 0,534 & 7,91 & 17. Sinto-me satisfeito com as minhas realizações. \\
\hline & 0,531 & 7,85 & 10. Eu tenho vontade de viver cada dia. \\
\hline & 0,482 & 7,02 & 7. Sinto-me realizado com o que faço. \\
\hline & 0,430 & 6,17 & 13. Eu gosto de estar na companhia de outras pessoas. \\
\hline & 0,410 & 5,86 & 5. Eu posso fazer as coisas que quero. \\
\hline & 0,387 & 5,50 & 16. Eu escolho fazer coisas que nunca fiz antes. \\
\hline & 0,383 & 5,44 & 3. Eu me sinto livre para planejar o futuro. \\
\hline \multirow[t]{6}{*}{2} & 0,699 & 8,11 & 1. Minha idade me impede de fazer as coisas que gostaria. \\
\hline & 0,596 & 6,53 & 8. Minha saúde me impede de fazer as coisas que quero. \\
\hline & 0,491 & 9,64 & 4. Eu me sinto deixado de lado. \\
\hline & 0,450 & 5,94 & 2. Eu sinto que não tenho controle sobre o que acontece comigo. \\
\hline & 0,429 & 5,64 & 6. As responsabilidades familiares me impedem de fazer o que quero. \\
\hline & 0,396 & 5,17 & 9. A falta de dinheiro me impede de fazer as coisas que quero. \\
\hline
\end{tabular}

Nota: medidas de adequação de ajuste obtidas: qui-quadrado = 433,37; qui-quadrado DF = 151; valor de $p<0,0001$; qui-quadrado ratio = 2,87; Goodness of Fit Index $(\mathrm{GFI})=0,8 ; \mathrm{GFI}$ Adjusted for Degrees of Freedom $(\mathrm{AGFI})=0,7606 ;$ Bentler's Comparative Fit Index $(\mathrm{CFI})=0,7241$; Bentler \& Bonett's $26(\mathrm{NNFI})=0.6876$; Standardized Root Mean Square Residual $(\mathrm{SRMR})=0.0902 ;$ RMSEA estimate $=0,0928 / \mathrm{IC} 90 \%: 0,0827-0,1031$.

Medidas de adequação de ajuste esperadas: valor de $p$ qui-quadrado $>0,05$; razão qui-quadrado $<2,0 ; \mathrm{GFI} \geq 0,85 ; \mathrm{AGFI} \geq$ 0,80; $\mathrm{CFI} \geq 0,90 ; \mathrm{NNFI} \geq 0,90 ;$ SRMR $\leq 0,10 ; \mathrm{RMSEA} \leq 0,0826$

* Valores significativos das cargas padronizadas dos itens $(p<0,05)$ para $t>1,9626$. Itens por domínio na CASP original: controle: 1 a 4; autonomia: 5 a 9; prazer: 10 a 14 e autorrealização: 15 a 19.

ausência de correlação entre o item 4 da escala de satisfação ("Dentro do possível, tenho conseguido as coisas importantes que quero da vida") e os itens 3, 4, 5 e 10 da CASP-19; entre o item 5 da escala de satisfação ("Se pudesse viver uma segunda vez, não mudaria quase nada na minha vida”) e os itens 3, 4 e 5 da CASP-19 (Figura 1).

Foram feitas análises de consistência interna de todos os instrumentos escalares para a amostra como um todo e para grupos estratificados por anos de escolaridade e por idade. Para a análise da CASP-19, foram considerados os itens da escala como um todo, os itens dos quatro fatores originais e os das soluções com três ou dois fatores obtidas por meio das AFE a AGC.

A CASP alcançou valores $\alpha$ de Cronbach maiores do que as escalas de satisfação e de felicidade subjetiva em todos os estratos considerados. Teve valores ligeiramente mais altos no grupo de 55 anos e mais do que no de 44-54 anos. Os índices $\alpha$ da escala de felicidade foram baixos, indicando relativamente baixa consistência interna, de forma oposta ao observado com as outras duas escalas. Os domínios controle e autonomia alcançaram níveis mais baixos de consistência interna do que os domínios autorrealização e prazer. A retirada de itens desses domínios mostrou-se responsável por alguma melhoria dos índices $\alpha$. As respostas dos segmentos mais escolarizados e com menor idade mostraram-se mais consistentes do que as dos menos escolarizados e as dos mais velhos. 
Figura 1

Correlações entre os escores totais na CASP-19 e na Escala de Felicidade Subjetiva e entre os escores totais na CASP-19 e na Escala de Satisfação com a Vida $(\mathrm{n}=368)$. CASP-19, Brasil, 2018.

1a) Escala de Satisfação com a Vida

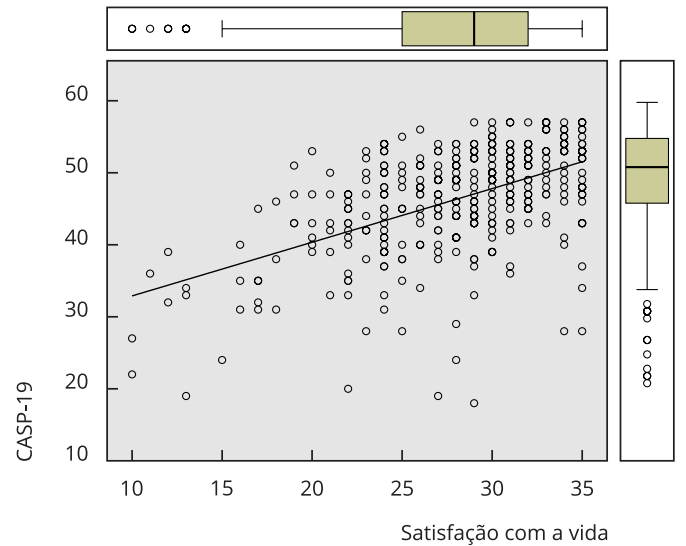

2b) Escala de Felicidade Subjetiva

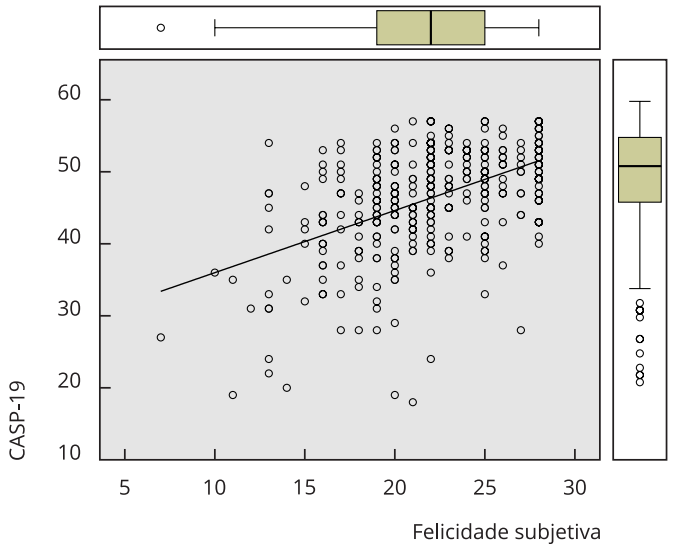

As AFE e AFC resultaram em estruturas de três e de dois fatores nas quais o fator 1 (autorrealização e prazer) alcançou altos valores de consistência interna, e os fatores 2 e 3 alcançaram índices intermediários, mas aceitáveis (Tabela 3).

\section{Fase 4}

Não foram observadas diferenças estatisticamente significativas entre as pontuações totais dos grupos de sexo, idade, escolaridade e status conjugal na CASP-19. Entretanto, os homens pontuaram significativamente mais alto do que as mulheres nos itens 12 e 14, que têm conotação de satisfação. Os idosos mais velhos pontuaram significantemente mais alto nos itens 4 e 9 , denotativos de perda de autonomia com o envelhecimento, e 14 e 17, envolvendo senso de realização com a vida. Os de 60-74 anos pontuaram significativamente mais alto do que os de 44-59 no item 12 (satisfação). Os com 1-8 anos de escolaridade pontuaram mais alto do que os com 9 anos ou mais, nos itens 4 (isolamento) e 12 (satisfação); os com 9 ou mais anos de escolaridade pontuaram mais alto do que os com 1-8 anos no item 9 (restrição à autonomia por falta de dinheiro). Os participantes sem vida conjugal pontuaram significantemente mais alto do que os casados nos itens 5 e 6 (autonomia) e 15 (energia) (Tabela 4).

\section{Discussão}

Aplicada em 368 adultos e idosos residentes nas regiões Sudeste, Sul e Nordeste do Brasil, a CASP-19 foi explicada por dois fatores, o primeiro com itens dos domínios autorrealização e prazer e o segundo com itens de controle e autonomia, responsáveis por $42,3 \%$ da variância total da escala. O primeiro alcançou alto nível de consistência interna, e o segundo, nível intermediário. Valores mais altos de consistência interna foram obtidos no segmento com 9 ou mais anos de escolaridade do que no grupo de 1-8 anos, sem relações com idade cronológica. A CASP-19 covariou com escalas de satisfação e de felicidade subjetiva. Os homens e os de 44-74 anos pontuaram mais alto em autorrealização/prazer do que as mulheres e os de 75 anos e mais; os mais escolarizados, mais negativamente em controle/ 
Tabela 3

Indicadores de consistência interna da CASP-19, da escala de felicidade subjetiva e da escala de satisfação com a vida, considerando as variáveis escolaridade e idade. CASP-19, Brasil, 2016.

\begin{tabular}{|c|c|c|c|c|}
\hline Escala/fator/número de itens & $\begin{array}{l}\text { Coeficiente a } \\
\text { de Cronbach }\end{array}$ & $\begin{array}{l}\text { Itens com menor } \\
\text { consistência }\end{array}$ & $\begin{array}{l}\text { Correlação } \\
\text { com o total * }\end{array}$ & $\begin{array}{c}\text { Coeficiente } \alpha \\
\text { (após retirada) ** }\end{array}$ \\
\hline \multicolumn{5}{|l|}{ Amostra total $(n=368)$} \\
\hline CASP-19/Total & 0,850 & - & - & - \\
\hline CASP-19/Controle & 0,506 & $\mathrm{C3}$ & 0,164 & 0,508 \\
\hline CASP-19/Autonomia & 0,597 & A9 & 0,208 & 0,605 \\
\hline CASP-19/Prazer & 0,726 & - & - & - \\
\hline CASP-19/Autorrealização & 0,722 & - & - & - \\
\hline Felicidade/Total & 0,591 & F4 & 0,107 & 0,707 \\
\hline Satisfação/Total & 0,808 & - & - & - \\
\hline \multicolumn{5}{|c|}{9 ou mais anos de escolaridade $(n=246)$} \\
\hline CASP-19/Total & 0,874 & - & - & - \\
\hline CASP-19/Controle & 0,552 & - & - & - \\
\hline CASP-19/Autonomia & 0,645 & - & - & - \\
\hline CASP-19/Prazer & 0,767 & - & - & - \\
\hline CASP-19/Autorrealização & 0,766 & - & - & - \\
\hline Felicidade/Total & 0,631 & $\mathrm{~F} 4$ & 0,160 & 0,727 \\
\hline Satisfação/Total & 0,851 & - & - & - \\
\hline \multicolumn{5}{|l|}{$1-8$ anos de escolaridade $(n=122)$} \\
\hline CASP-19/Total & 0,793 & - & - & - \\
\hline CASP-19/Controle & 0,431 & $\mathrm{C} 3$ & 0,042 & 0,480 \\
\hline \multirow[t]{2}{*}{ CASP-19/Autonomia } & 0,492 & A7 & 0,053 & 0,568 \\
\hline & & A9 & 0,192 & 0,617 \\
\hline CASP-19/Prazer & 0,616 & - & - & - \\
\hline CASP-19/Autorrealização & 0,628 & - & - & - \\
\hline Felicidade/Total & 0,510 & $\mathrm{~F} 4$ & 0,019 & 0,666 \\
\hline Satisfação/Total & 0,712 & - & - & - \\
\hline \multicolumn{5}{|l|}{ Idade: 55 anos e mais $(n=337)$} \\
\hline CASP-19/Total & 0,851 & - & - & - \\
\hline \multirow[t]{2}{*}{ CASP-19/Controle } & 0,496 & $\mathrm{C} 3$ & 0,146 & 0,504 \\
\hline & & A5 & 0,107 & 0,551 \\
\hline \multirow[t]{2}{*}{ CASP-19/Autonomia } & 0,580 & A9 & 0,190 & 0,602 \\
\hline & & A8 & 0,104 & 0,724 \\
\hline CASP-19/Prazer & 0,731 & - & - & - \\
\hline CASP-19/Autorrealização & 0,731 & - & - & - \\
\hline Felicidade/Total & 0,586 & $\mathrm{~F} 4$ & 0,092 & 0,711 \\
\hline Satisfação/Total & 0,815 & - & - & - \\
\hline \multicolumn{5}{|l|}{ Idade: $44-54$ anos $(n=30)$} \\
\hline CASP-19/Total & 0,849 & $\mathrm{C3}$ & 0,098 & 0,857 \\
\hline CASP-19/Controle & 0,644 & $\mathrm{C} 1$ & 0,200 & 0,663 \\
\hline CASP-19/Autonomia & 0,758 & - & - & - \\
\hline CASP-19/Prazer & 0,662 & - & - & - \\
\hline CASP-19/Autorrealização & 0,584 & S17 & 0,151 & 0,670 \\
\hline Felicidade/Total & 0,637 & $\mathrm{~F} 4$ & 0,155 & 0,738 \\
\hline Satisfação/Total & 0,751 & - & - & - \\
\hline \multicolumn{5}{|l|}{ 1a subamostra $(n=150)[A F E]$} \\
\hline CASP-19/Fator 1 & 0,893 & - & - & - \\
\hline CASP-19/Fator 2 & 0,620 & - & - & - \\
\hline CASP-19/Fator 3 & 0,650 & - & - & - \\
\hline \multicolumn{5}{|l|}{ 1ạ subamostra $(n=150)[A F E]$} \\
\hline CASP-19/Fator 1 & 0,887 & - & - & - \\
\hline CASP-19/Fator 2 & 0,620 & - & - & - \\
\hline \multicolumn{5}{|l|}{ 2a subamostra $(n=218)[A F C]$} \\
\hline CASP-19/Fator 1 & 0,837 & - & - & - \\
\hline CASP-19/Fator 2 & 0,670 & - & - & - \\
\hline
\end{tabular}

AFC: análises fatoriais confirmatórias; AFE: análises fatoriais exploratórias (AFE).

Nota: itens invertidos: CASP C1, C2, C4, A6, A8, A9; Felicidade: F1 e F4.

* Correlação do item com o total do respectivo domínio, sem considerar o item no escore total;

** Coeficiente a de Cronbach após retirada consecutiva dos itens com menor consistência. 
Tabela 4

Médias e desvios-padrão da amostra na CASP-19, por sexo, idade, escolaridade e status conjugal. Brasil, 2016.

\begin{tabular}{|c|c|c|c|c|c|c|c|c|c|}
\hline \multirow[t]{2}{*}{ Itens } & \multicolumn{2}{|c|}{ Sexo } & \multicolumn{3}{|c|}{ Idade } & \multicolumn{2}{|c|}{ Anos de escolaridade } & \multicolumn{2}{|c|}{ Casados/Companheiro } \\
\hline & $\begin{array}{c}\text { Masculino } \\
(\mathrm{n}=52)\end{array}$ & $\begin{array}{c}\text { Feminino } \\
(n=316)\end{array}$ & $\begin{array}{c}44-59 \\
(n=86)\end{array}$ & $\begin{array}{c}60-74 \\
(n=230)\end{array}$ & $\begin{array}{c}75+ \\
(n=51)\end{array}$ & $\begin{array}{c}1-8 \\
(n=122)\end{array}$ & $\begin{array}{c}9 e+ \\
(n=246)\end{array}$ & $\begin{array}{c}\operatorname{Sim} \\
(n=168)\end{array}$ & $\begin{array}{c}\text { Não } \\
(n=197)\end{array}$ \\
\hline 1. Minha idade me impede de & \multicolumn{2}{|c|}{$p=0,627$} & \multicolumn{3}{|c|}{$p=0,124$} & \multicolumn{2}{|c|}{$p=702$} & \multicolumn{2}{|c|}{$p=0,922$} \\
\hline fazer as coisas que eu gostaria. & $\begin{array}{l}2,46 \\
(0,75)\end{array}$ & $\begin{array}{c}2,45 \\
(0,67)\end{array}$ & $\begin{array}{c}2,55 \\
(0,62)\end{array}$ & $\begin{array}{c}2,44 \\
(0,69)\end{array}$ & $\begin{array}{c}2,31 \\
(0,70)\end{array}$ & $\begin{array}{l}2,41 \\
(0,74)\end{array}$ & $\begin{array}{c}2,47 \\
(0,64)\end{array}$ & $\begin{array}{c}2,45 \\
(0,69)\end{array}$ & $\begin{array}{l}2,45 \\
(0,67)\end{array}$ \\
\hline 2. Sinto que não tenho & \multicolumn{2}{|c|}{$p=0,141$} & \multicolumn{3}{|c|}{$p=0,448$} & \multicolumn{2}{|c|}{$p=0,242$} & \multicolumn{2}{|c|}{$p=0,155$} \\
\hline $\begin{array}{l}\text { controle sobre o que acontece } \\
\text { comigo. }\end{array}$ & $\begin{array}{c}2,46 \\
(0,93)\end{array}$ & $\begin{array}{c}2,37 \\
(0,83)\end{array}$ & $\begin{array}{c}2,37 \\
(0,81)\end{array}$ & $\begin{array}{c}2,37 \\
(0,86)\end{array}$ & $\begin{array}{c}2,47 \\
(0,88)\end{array}$ & $\begin{array}{c}2,27 \\
(0,97)\end{array}$ & $\begin{array}{c}2,44 \\
(0,77)\end{array}$ & $\begin{array}{c}2,33 \\
(0,87)\end{array}$ & $\begin{array}{c}2,44 \\
(0,81)\end{array}$ \\
\hline 3. Sinto-me livre para planejar & \multicolumn{2}{|c|}{$p=0,169$} & \multicolumn{3}{|c|}{$p=0,558$} & \multicolumn{2}{|c|}{$p=0,973$} & \multicolumn{2}{|c|}{$p=0,488$} \\
\hline o futuro. & $\begin{array}{c}2,37 \\
(1,00)\end{array}$ & $\begin{array}{c}2,22 \\
(1,04)\end{array}$ & $\begin{array}{l}2,36 \\
(0,95)\end{array}$ & $\begin{array}{c}2,23 \\
(1,04)\end{array}$ & $\begin{array}{c}2,08 \\
(1,19)\end{array}$ & $\begin{array}{c}2,23 \\
(1,08)\end{array}$ & $\begin{array}{c}2,25 \\
(1,03)\end{array}$ & $\begin{array}{c}2,23 \\
(1,00)\end{array}$ & $\begin{array}{c}2,25 \\
(1,09)\end{array}$ \\
\hline \multirow[t]{2}{*}{ 4. Sinto-me deixado de lado. } & \multicolumn{2}{|c|}{$p=0,101$} & \multicolumn{3}{|c|}{$p=0,004$} & \multicolumn{2}{|c|}{$p=0,039$} & \multicolumn{2}{|c|}{$p=0,130$} \\
\hline & $\begin{array}{l}2,67 \\
(0,64)\end{array}$ & $\begin{array}{l}2,50 \\
(0,76)\end{array}$ & $\begin{array}{l}2,47 \\
(0,74)\end{array}$ & $\begin{array}{l}2,49 \\
(0,76)\end{array}$ & $\begin{array}{l}2,78 \\
(0,64)\end{array}$ & $\begin{array}{l}2,57 \\
(0,84)\end{array}$ & $\begin{array}{l}2,50 \\
(0,70)\end{array}$ & $\begin{array}{l}2,60 \\
(0,65)\end{array}$ & $\begin{array}{l}2,46 \\
(0,82)\end{array}$ \\
\hline \multirow{2}{*}{$\begin{array}{l}\text { 5. Eu consigo fazer as coisas } \\
\text { que quero. }\end{array}$} & \multicolumn{2}{|c|}{$p=0,588$} & \multicolumn{3}{|c|}{$P=0,753$} & \multicolumn{2}{|c|}{$p=0,291$} & \multicolumn{2}{|c|}{$p<0,006$} \\
\hline & $\begin{array}{c}2,10 \\
(1,00)\end{array}$ & $\begin{array}{l}2,29 \\
(0,88)\end{array}$ & $\begin{array}{c}2,36 \\
(0,71)\end{array}$ & $\begin{array}{l}2,23 \\
(0,95)\end{array}$ & $\begin{array}{c}2,31 \\
(0,94)\end{array}$ & $\begin{array}{c}2,32 \\
(0,92)\end{array}$ & $\begin{array}{l}2,25 \\
(0,89)\end{array}$ & $\begin{array}{c}2,14 \\
(0,94)\end{array}$ & $\begin{array}{c}2,39 \\
(0,85)\end{array}$ \\
\hline 6. As responsabilidades & $p=0$ & ,994 & & $p=0,014$ & & $p=$ & 111 & $\mathrm{p}<$ & 001 \\
\hline $\begin{array}{l}\text { familiares me impedem de } \\
\text { fazer o que quero. }\end{array}$ & $\begin{array}{c}2,33 \\
(0,87)\end{array}$ & $\begin{array}{l}2,36 \\
(0,77)\end{array}$ & $\begin{array}{l}2,27 \\
(0,74)\end{array}$ & $\begin{array}{c}2,35 \\
(0,81)\end{array}$ & $\begin{array}{l}2,57 \\
(0,78)\end{array}$ & $\begin{array}{c}2,42 \\
(0,83)\end{array}$ & $\begin{array}{c}2,33 \\
(0,77)\end{array}$ & $\begin{array}{l}2,23 \\
(0,80)\end{array}$ & $\begin{array}{c}2,47 \\
(0,76)\end{array}$ \\
\hline 7. Sinto-me realizado com o & $p=0$ & ,349 & & $p=0,060$ & & $p=$ & 660 & & 980 \\
\hline que faço. & $\begin{array}{c}2,56 \\
(0,82)\end{array}$ & $\begin{array}{c}2,47 \\
(0,83)\end{array}$ & $\begin{array}{c}2,36 \\
(0,82)\end{array}$ & $\begin{array}{c}2,52 \\
(0,80)\end{array}$ & $\begin{array}{c}2,53 \\
(0,90)\end{array}$ & $\begin{array}{c}2,54 \\
(0,74)\end{array}$ & $\begin{array}{c}2,46 \\
(0,86)\end{array}$ & $\begin{array}{c}2,48 \\
(0,84)\end{array}$ & $\begin{array}{c}2,49 \\
(0,82)\end{array}$ \\
\hline 8. Minha saúde me impede de & $p=0$ & ,753 & & $p=0,720$ & & $p=$ & 136 & $p=$ & 815 \\
\hline fazer as coisas que quero. & $\begin{array}{c}2,38 \\
(0,79)\end{array}$ & $\begin{array}{c}2,36 \\
(0,77)\end{array}$ & $\begin{array}{c}2,38 \\
(0,75)\end{array}$ & $\begin{array}{c}2,37 \\
(0,78)\end{array}$ & $\begin{array}{c}2,29 \\
(0,78)\end{array}$ & $\begin{array}{c}2,25 \\
(0,86)\end{array}$ & $\begin{array}{c}2,42 \\
(0,71)\end{array}$ & $\begin{array}{c}2,37 \\
(0,74)\end{array}$ & $\begin{array}{c}2,37 \\
(0,79)\end{array}$ \\
\hline 9. A falta de dinheiro me & $p=0$ & ,298 & & $p=0,026$ & & $\mathrm{p}<$ & 001 & $p=$ & 831 \\
\hline $\begin{array}{l}\text { impede de fazer as coisas que } \\
\text { quero. }\end{array}$ & $\begin{array}{c}2,02 \\
(0,89)\end{array}$ & $\begin{array}{c}1,87 \\
(0,93)\end{array}$ & $\begin{array}{c}1,67 \\
(0,92)\end{array}$ & $\begin{array}{c}1,93 \\
(0,93)\end{array}$ & $\begin{array}{c}2,06 \\
(0,85)\end{array}$ & $\begin{array}{c}1,58 \\
(1,03)\end{array}$ & $\begin{array}{c}2,05 \\
(0,83)\end{array}$ & $\begin{array}{c}1,91 \\
(0,94)\end{array}$ & $\begin{array}{c}1,89 \\
(0,91)\end{array}$ \\
\hline 10. Eu tenho vontade de viver & $p=0$ & ,722 & & $p=0,544$ & & $p=$ & 063 & $p=$ & 482 \\
\hline cada dia. & $\begin{array}{c}2,88 \\
(0,37)\end{array}$ & $\begin{array}{c}2,83 \\
(0,56)\end{array}$ & $\begin{array}{c}2,81 \\
(0,52)\end{array}$ & $\begin{array}{c}2,84 \\
(0,54)\end{array}$ & $\begin{array}{c}2,84 \\
(0,61)\end{array}$ & $\begin{array}{c}2,90 \\
(0,41)\end{array}$ & $\begin{array}{c}2,80 \\
(0,59)\end{array}$ & $\begin{array}{c}2,86 \\
(0,48)\end{array}$ & $\begin{array}{c}2,81 \\
(0,58)\end{array}$ \\
\hline 11. Eu sinto que minha vida & $p=0$ &, 223 & & $p=0,185$ & & $p=$ & 346 & & 703 \\
\hline tem sentido. & $\begin{array}{c}2,88 \\
(0,37)\end{array}$ & $\begin{array}{c}2,76 \\
(0,63)\end{array}$ & $\begin{array}{c}2,76 \\
(0,59)\end{array}$ & $\begin{array}{c}2,77 \\
(0,61)\end{array}$ & $\begin{array}{c}2,84 \\
(0,64)\end{array}$ & $\begin{array}{c}2,80 \\
(0,58)\end{array}$ & $\begin{array}{c}2,76 \\
(0,62)\end{array}$ & $\begin{array}{c}2,79 \\
(0,59)\end{array}$ & $\begin{array}{c}2,76 \\
(0,63)\end{array}$ \\
\hline 12. Eu gosto das coisas que & $p=0$ &, 051 & & $p=0,005$ & & $p=$ & 011 & & 917 \\
\hline faço. & $\begin{array}{c}2,87 \\
(0,39)\end{array}$ & $\begin{array}{c}2,69 \\
(0,62)\end{array}$ & $\begin{array}{c}2,58 \\
(0,62)\end{array}$ & $\begin{array}{c}2,76 \\
(0,57)\end{array}$ & $\begin{array}{c}2,76 \\
(0,65)\end{array}$ & $\begin{array}{c}2,83 \\
(0,45)\end{array}$ & $\begin{array}{c}2,66 \\
(0,65)\end{array}$ & $\begin{array}{c}2,71 \\
(0,61)\end{array}$ & $\begin{array}{c}2,72 \\
(0,59)\end{array}$ \\
\hline 13. Eu gosto de estar na & $p=0$ & ,344 & & $p=0,211$ & & $p=$ & 218 & & 680 \\
\hline companhia de outras pessoas. & $\begin{array}{c}2,77 \\
(0,42)\end{array}$ & $\begin{array}{c}2,69 \\
(0,67)\end{array}$ & $\begin{array}{c}2,62 \\
(0,53)\end{array}$ & $\begin{array}{c}2,67 \\
(0,65)\end{array}$ & $\begin{array}{c}2,61 \\
(0,75)\end{array}$ & $\begin{array}{c}2,71 \\
(0,56)\end{array}$ & $\begin{array}{c}2,62 \\
(0,67)\end{array}$ & $\begin{array}{c}2,67 \\
(0,61)\end{array}$ & $\begin{array}{c}2,63 \\
(0,67)\end{array}$ \\
\hline 14. Quando avalio a minha & $p=0$ &, 032 & & $p=0,023$ & & $p=$ & 060 & & 877 \\
\hline vida até aqui, eu me sinto feliz. & $\begin{array}{c}2,67 \\
(0,58)\end{array}$ & $\begin{array}{c}2,44 \\
(0,75)\end{array}$ & $\begin{array}{c}2,40 \\
(0,75)\end{array}$ & $\begin{array}{c}2,45 \\
(0,75)\end{array}$ & $\begin{array}{c}2,71 \\
(0,61)\end{array}$ & $\begin{array}{c}2,45 \\
(0,74)\end{array}$ & $\begin{array}{c}2,48 \\
(0,73)\end{array}$ & $\begin{array}{c}2,48 \\
(0,74)\end{array}$ & $\begin{array}{c}2,47 \\
(0,73)\end{array}$ \\
\hline
\end{tabular}

(continua) 
Tabela 4 (continuação)

\begin{tabular}{|c|c|c|c|c|c|c|c|c|c|}
\hline \multirow[t]{2}{*}{ Itens } & \multicolumn{2}{|c|}{ Sexo } & \multicolumn{3}{|c|}{ Idade } & \multicolumn{2}{|c|}{ Anos de escolaridade } & \multicolumn{2}{|c|}{ Casados/Companheiro } \\
\hline & $\begin{array}{c}\text { Masculino } \\
\quad(n=52)\end{array}$ & $\begin{array}{c}\text { Feminino } \\
(n=316)\end{array}$ & $\begin{array}{c}44-59 \\
(n=86)\end{array}$ & $\begin{array}{c}60-74 \\
(n=230)\end{array}$ & $\begin{array}{c}75+ \\
(n=51)\end{array}$ & $\begin{array}{c}1-8 \\
(n=122)\end{array}$ & $\begin{array}{c}9 e+ \\
(n=246)\end{array}$ & $\begin{array}{c}\text { Sim } \\
(n=168)\end{array}$ & $\begin{array}{c}\text { Não } \\
(n=197)\end{array}$ \\
\hline 15. Ultimamente, eu tenho me & \multicolumn{2}{|c|}{$p=0,997$} & \multicolumn{3}{|c|}{$p=0,080$} & \multicolumn{2}{|c|}{$p=0,062$} & \multicolumn{2}{|c|}{$p=0,041$} \\
\hline sentido cheio de energia. & $\begin{array}{c}2,40 \\
(0,72)\end{array}$ & $\begin{array}{c}2,39 \\
(0,75)\end{array}$ & $\begin{array}{c}2,28 \\
(0,79)\end{array}$ & $\begin{array}{c}2,45 \\
(0,73)\end{array}$ & $\begin{array}{c}2,31 \\
(0,70)\end{array}$ & $\begin{array}{c}2,36 \\
(0,81)\end{array}$ & $\begin{array}{c}2,41 \\
(0,71)\end{array}$ & $\begin{array}{c}2,30 \\
(0,79)\end{array}$ & $\begin{array}{c}2,47 \\
(0,70)\end{array}$ \\
\hline 16. Eu escolho fazer coisas que & \multicolumn{2}{|c|}{$p=0,672$} & \multicolumn{3}{|c|}{$p=0,939$} & \multicolumn{2}{|c|}{$p=0,060$} & \multicolumn{2}{|c|}{$p=0,749$} \\
\hline nunca fiz antes. & $\begin{array}{l}1,81 \\
(1,0)\end{array}$ & $\begin{array}{c}1,90 \\
(0,87)\end{array}$ & $\begin{array}{c}1,92 \\
(0,88)\end{array}$ & $\begin{array}{c}1,87 \\
(0,88)\end{array}$ & $\begin{array}{c}1,86 \\
(1,00)\end{array}$ & $\begin{array}{c}1,75 \\
(1,01)\end{array}$ & $\begin{array}{c}1,95 \\
(0,83)\end{array}$ & $\begin{array}{c}1,87 \\
(0,89)\end{array}$ & $\begin{array}{c}1,90 \\
(0,89)\end{array}$ \\
\hline 17. Eu me sinto satisfeito com & \multicolumn{2}{|c|}{$p<0,067$} & \multicolumn{3}{|c|}{$p<0,001$} & \multicolumn{2}{|c|}{$p=0,062$} & \multicolumn{2}{|c|}{$p=0,489$} \\
\hline o rumo da minha vida. & $\begin{array}{c}2,71 \\
(0,53)\end{array}$ & $\begin{array}{c}2,53 \\
(0,68)\end{array}$ & $\begin{array}{c}2,34 \\
(0,80)\end{array}$ & $\begin{array}{c}2,59 \\
(0,60)\end{array}$ & $\begin{array}{c}2,76 \\
(0,61)\end{array}$ & $\begin{array}{c}2,62 \\
(0,67)\end{array}$ & $\begin{array}{c}2,52 \\
(0,66)\end{array}$ & $\begin{array}{c}2,54 \\
(0,63)\end{array}$ & $\begin{array}{c}2,56 \\
(0,70)\end{array}$ \\
\hline 18. Eu sinto que a vida & \multicolumn{2}{|c|}{$p=0,414$} & \multicolumn{3}{|c|}{$p=0,435$} & \multicolumn{2}{|c|}{$p=0,510$} & \multicolumn{2}{|c|}{$p=0,091$} \\
\hline $\begin{array}{l}\text { me proporciona muitas } \\
\text { oportunidades. }\end{array}$ & $\begin{array}{c}2,37 \\
(0,76)\end{array}$ & $\begin{array}{c}2,44 \\
(0,77)\end{array}$ & $\begin{array}{c}2,34 \\
(0,80)\end{array}$ & $\begin{array}{c}2,44 \\
(0,77)\end{array}$ & $\begin{array}{c}2,51 \\
(0,70)\end{array}$ & $\begin{array}{c}2,46 \\
(0,76)\end{array}$ & $\begin{array}{c}2,41 \\
(0,77)\end{array}$ & $\begin{array}{c}2,36 \\
(0,78)\end{array}$ & $\begin{array}{c}2,48 \\
(0,76)\end{array}$ \\
\hline 19. Eu acho que o futuro & \multicolumn{2}{|c|}{$p=0,992$} & \multicolumn{3}{|c|}{$p=0,691$} & \multicolumn{2}{|c|}{$p=0,666$} & \multicolumn{2}{|c|}{$p=0,561$} \\
\hline reserva coisas boas para mim. & $\begin{array}{l}2,56 \\
(0,80)\end{array}$ & $\begin{array}{l}2,610 \\
(0,69)\end{array}$ & $\begin{array}{c}2,65 \\
(0,62)\end{array}$ & $\begin{array}{l}2,58 \\
(0,73)\end{array}$ & $\begin{array}{c}2,63 \\
(0,77)\end{array}$ & $\begin{array}{c}2,61 \\
(0,73)\end{array}$ & $\begin{array}{c}2,60 \\
(0,70)\end{array}$ & $\begin{array}{l}2,60 \\
(0,67)\end{array}$ & $\begin{array}{c}2,61 \\
(0,74)\end{array}$ \\
\hline
\end{tabular}

* Testes U de Mann-Whitney para comparação de duas amostras independentes e Kruskal-Wallis para comparação de três amostras independentes; $p \leq$ 0.05. Itens por domínio na CASP-19 original: controle: 1 a 4; autonomia: 5 a 9; prazer: 10 a 14 e autorrealização: 15 a 19.

autonomia e mais positivamente em autorrealização/prazer do que os participantes de 44-74 anos e os menos escolarizados; os que viviam sozinhos, mais positivamente em autonomia/controle do que os casados.

As soluções fatoriais obtidas neste estudo sugerem que a escala é descrita melhor como medida de bem-estar psicológico ou de ajustamento psicológico na vida adulta e na velhice, no sentido eudaimônico 19,20,21,22,23,24,25,26,27,28,29,30, do que a medida de autorrelato sobre experiências de controle, autonomia autorrealização e prazer. Dos itens que compõem o fator 1, pode ser abstraída a seguinte ideia: perceber que minha vida tem sentido, que gosto das coisas que faço e que me sinto realizado estimula-me a prosseguir, traz-me satisfação e ajuda-me a ter boas expectativas para o futuro. As palavraschave desse fator são significado e satisfação, não prazer no sentido hedônico. O raciocínio subjacente ao fator 2 é: percebo que não tenho mais a mesma autonomia nem a mesma possibilidade de controlar o ambiente que tinha antes. $\mathrm{O}$ item 16, classificado pelos autores ingleses como de autorrealização, aparece nesse fator, com conotação de autonomia. A primazia dos domínios autorrealização e prazer sobre os domínios controle e autonomia não foi observada em outros estudos psicométricos com a CASP-19, nem mesmo no de Lima et al. ${ }^{20}$, que replicou a estrutura em quatro fatores. Faz-se necessária a realização de novas investigações para testar esses resultados. No entanto, no estudo de Lima et al. 20 e no nosso, o domínio prazer apresentou-se com baixa relação com os demais, fortalecendo a ideia de que a CASP-19 é medida de bem-estar eudaimônico.

Os resultados das análises de consistência interna e de construto para a CASP-19 sugerem a existência de uma dificuldade de origem cultural e educacional para lidar simultaneamente com a presença de itens positivos e negativos. Havia assimetria entre os itens, pois, para obter alta pontuação nos itens positivos, o participante devia responder "sempre", ao passo que, para obter pontos positivos nos itens negativos, deveria responder "nunca" ou "raramente". Na escala de felicidade subjetiva, o problema é maior, porque existe assimetria entre o enunciado e as alternativas de três dos seus quatro itens, assimetria essa que terá afetado a consistência interna e a força da correlação com a CASP19. São reconhecidos os efeitos desses eventos sobre a consistência interna dos instrumentos 12 . A hipótese do efeito da redação dos itens sobre as respostas é fortalecida pelos baixos valores de $\alpha$ observados na escala de felicidade, das baixas correlações entre os itens dessa escala e os da CASP-19 e da predominância de itens de controle e autonomia (os negativos, invertidos na análise de dados), 
entre os que provocariam melhoria nos indicadores de consistência interna, caso fossem excluídos. Além disso, entre os participantes com 9 ou mais anos de escolaridade, foram observados valores mais elevados de consistência interna nas três escalas do que no grupo com 1-8 anos de educação formal.

Em combinação com suportes visuais esclarecedores do significado da gradação das respostas e do significado das âncoras das escalas, instruções que mencionam frequência de ocorrência de sentimentos, percepções ou avaliações podem contribuir para a melhora da eficácia dos instrumentos, principalmente para indivíduos com baixa escolaridade e com dificuldade de compreender o fraseado da escala ou a natureza da tarefa a realizar. A substituição do formato escrito pelo de entrevista, combinada a suportes eficazes, pode ser uma boa solução para essas questões. De todo modo, recomendase cautela com a aplicação da CASP-19 em adultos e idosos com baixa escolaridade.

A escolha das escalas de satisfação com a vida 27 e de felicidade subjetiva 28 para estabelecer os níveis de validade convergente da CASP-19 na amostra brasileira foi baseada no argumento de Hyde et al. ${ }^{1}$ de que, para cumprir esse objetivo, as medidas de qualidade de vida devem ser diferentes das que avaliam fenômenos contextuais e individuais que a influenciam, tais como a saúde, as redes de relações sociais e as circunstâncias materiais. Além disso, satisfação de necessidades básicas de controle, autonomia, autorrealização e prazer, satisfação com a vida e felicidade subjetiva são construtos aparentados, tanto que a Escala de Satisfação com a Vida 27 foi usada pelos autores ingleses já na primeira validação convergente da CASP-19 1.

Embora em sua maioria significativos, não foram altos os valores das correlações bivariadas entre esses instrumentos, possivelmente devido às dificuldades já mencionadas que os respondentes tiveram para lidar com a assimetria entre os itens 28. Em termos conceituais, é difícil admitir similaridade entre os itens da CASP-19 que envolvem percepção de controle e autonomia, os da escala de felicidade subjetiva, que requerem que os respondentes comparem sua felicidade com a de outrem e que se comparem com pessoas não muito felizes. A ausência de correlação entre os itens 4 ("Dentro do possível, tenho conseguido as coisas importantes que quero da vida") e 5 ("Se pudesse viver uma segunda vez, não mudaria quase nada na minha vida"), da escala de satisfação, e itens de controle e autonomia da CASP-19 pode ser igualmente explicada pelo seu pertencimento a universos diferentes (autorrealização na escala de satisfação x controle e autonomia na CASP-19). São motivos suficientes para recomendar cautela ou não adoção da escala de felicidade subjetiva em futuras investigações de validade convergente para a CASP-19.

Não foram observadas diferenças estatisticamente significativas entre as pontuações totais dos grupos de sexo, idade, escolaridade e status conjugal na CASP-19 por dois possíveis motivos. Em primeiro lugar, porque a maioria dos participantes pontuou alto no instrumento. Em segundo lugar, pelo tamanho relativamente pequeno da amostra. No entanto, dados de pesquisa dão conta da ocorrência de pontuação mais alta entre os homens do que entre as mulheres, nos grupos mais escolarizados do que nos menos escolarizados e entre os mais novos do que entre os mais velhos 31,32. No Brasil, Marques et al. 22 observaram que idosos de 60-69 anos pontuaram mais alto na CASP-19 do que os de 70 anos e mais, assim como os com 12 ou mais anos de escolaridade pontuaram mais alto do que os com 11 ou menos. No estudo ora relatado, as pontuações dos participantes sem vida conjugal, nos itens 5 (consigo fazer as coisas que quero) e 6 (responsabilidades familiares restringem a autonomia), falam da relação entre essa condição e o senso de autonomia. Esses dados são indicadores da validade ecológica da CASP-19 no grupo entrevistado.

Há dados de estudos populacionais segundo os quais a qualidade de vida indicada pela CASP-19 relaciona-se com nível socioeconômico e desigualdade socioeconômica, condições em que sexo, idade e escolaridade têm forte presença 4,13,18,31; incapacidade e inatividade geradas por doenças crônicas 32 e fragilidade ${ }^{29}$; rede de relações sociais e suporte social percebido 33 , e efeitos acumulativos de adversidades sofridas ao longo do curso de vida 34 . Embora a amostra deste estudo seja pequena e não representativa da população, os dados obtidos sugerem que a CASP-19 é sensível a essas condições, sugestão essa que será vantajosamente testada em futuras pesquisas populacionais brasileiras com grandes amostras.

Embora as conclusões e generalizações sejam limitadas pelo tamanho da amostra e pelo fato de ela ser de conveniência, este estudo oferece dois dados importantes ao conhecimento das características psicométricas da CASP-19, escala lastreada em um modelo psicológico de qualidade de vida. Um é relativo à observação de maior consistência interna no grupo de escolaridade mais alta, informação 
relevante a futuras utilizações da escala no contexto brasileiro, em que o nível educacional dos idosos é mais baixo do que o de países onde o instrumento tem sido utilizado. O outro se refere à primazia do fator autorrealização/prazer, esse um dado novo na literatura. Ambos merecem ser mais investigados em amostras maiores e probabilísticas.

A pesquisa é representativa da valorização de estudos de validação lastreados na teoria e na Estatística. As considerações críticas relativas ao conteúdo da CASP-19 estão alinhadas com o pensamento segundo o qual, até certo ponto, instrumentos psicométricos são uma obra em aberto. Seu uso em culturas diversas daquela de origem impõe desafios de natureza teórica e metodológica, cuja superação fortalece as teorias psicométricas e as próprias medidas, além de oferecer garantias da identificação de dados mais fidedignos.

\section{Colaboradores}

A. L. Neri planejou o estudo e coordenou sua realização, discutiu as análises estatísticas com F. S. A. Borim e escreveu a primeira versão do artigo. F. S. A. Borim realizou a discussão das análises estatísticas com A. L. Neri e revisou o artigo. S. S. T. Batistoni colaborou com a revisão de literatura e a revisão do artigo. M. Cachioni, D. F. Rabelo e A. P. Fontes realizaram a revisão da literatura e a revisão do artigo. M. S. Yassuda realizou a revisão do artigo. Todos os autores aprovaram a versão final a ser publicada.

\section{Agradecimentos}

Agradecimentos às instituições das cidades de Campinas, Hortolândia, Capivari e Taubaté (São Paulo), Maceió (Alagoas) e Passo Fundo (Rio Grande do Sul) por terem permitido e facilitado o acesso aos frequentadores de seus programas de educação não formal para idosos, para aplicação dos instrumentos utilizados no estudo.

\section{Referências}

1. Hyde M, Wiggins RD, Higgs P, Blane DB. A measure of quality of life in early old age: the theory, development and properties of a needs satisfaction model (CASP-19). Aging Ment Health 2003; 7:186-94.

2. Maslow AH. Toward a psychology of being. 2nd Ed. Princeton: Van Nostrand; 1968.

3. Gunnel D, Davey-Smith G, Frankel S, Nanchahal K, Braddon FM, Peters T. Life course exposure and later diseases: a follow-up study based on a survey of family diet and health in prewar Britain (1937-1939). Public Health 1996; 110:85-94.

4. Marmot M, Banks J, Blundell R, Lessof C, Nazroo J. Health, wealth and life styles of the older population in England: the 2002 English Longitudinal Study of Ageing. London: Institute for Fiscal Studies; 2002.

5. Taylor M, Brice J, Buck N, Prentice-Lane E. British household panel survey user manual. Volume A. Introduction, Technical Report and Appendices. Colchester: UK Data Archive, University of Essex; 2003.

6. Neugarten BL, Havighurst RJ, Tobin SS. The measurement of life satisfaction. J Gerontol 1961; 16:134-43.

7. Wiggins RD, Netuveli G, Hyde M, Higgs P, Blane D. The evaluation of a self-enumerated scale of quality of life (CASP-19) in the context of research on ageing: a combination of exploratory and confirmatory approaches. Soc Indic Res 2008; 89:61-77. 
8. Hamren K, Chungkham HS, Hyde M. Religion, spirituality, social support and quality of life: measurement and predictors CASP-12(v2) amongst older Ethiopians living in Addis Ababa. Aging Ment Health 2015; 19:610-21.

9. Kim GR, Netuveli G, Blane D, Peasey A, Malyutina S, Simonova G, et al. Psychometric properties and confirmatory factor analysis of the CASP-19, a measure of quality of life in early old age: the HAPIEE study. Aging Ment Health 2015; 19:595-609.

10. Oluboyede Y, Smith AB. Evidence for a unidimensional 15-item version of the CASP-19 using a Rasch model approach. Qual Life Res 2013; 22:2429-33.

11. Sexton E, King-Kallimanis BL, Conroy RM, Hickey A. Psychometric evaluation of the CASP-19 quality of life scale in an older Irish cohort. Qual Life Res 2013; 22:2549-59.

12. Sim J, Bartlam B, Bernard M. The CASP-19 as a measure of quality of life in old age: evaluation of its use in a retirement community. Qual Life Res 2011; 20:997-1004.

13. Layte R, Sexton E, Savva G. Quality of life in older age: evidence from an Irish cohort study. J Am Geriatr Soc 2013; 61 Suppl 2:S299-305.

14. Wu TY, Chie WC, Kuo KL, Wong WK, Liu JP, Chiu ST, et al. Quality of life (QOL) among community dwelling older people in Taiwan measured by the CASP-19, an index to capture QOL in old age. Arch Gerontol Geriatr 2013; 57:143-50.

15. Sexton E, King-Kallimanis BL, Layte R, Hickey A. How does chronic disease status affect CASP quality of life at older ages? Examining the WHOICF disability domains as mediators of this relationship. Aging Ment Health 2015; 19:622-33.

16. National Institute on Aging. Growing older in America: the Health and Retiremnet Study. http://www.umich.edu/sitedocs/bro chure/HRS-brochure.pdf (acessado em 02/ Out/2017).

17. Towers A, Yeung P, Stevenson B, Stephens C, Alpass F. Quality of life in indigenous and non-indigenous older adults: assessing the CASP-12 factor structure and identifying a brief CASP-3. Qual Life Res 2015; 24:193-203.

18. Börsch-Supan A, Brugiavini A, Jürges $H$, Makenbach J, Siegrist J, Weber G. Health, ageing and retirement in Europe. First results from the survey of health, ageing and retirement in Europe (SHARE). Manneheim: Manneheim Research Institute for the Economics of Aging; 2005.

19. Hyde M, Higgs P, Wiggins RD, Blane D. A decade of research using the CASP scale: key findings and future directions. Aging Ment Health 2015 ; 19:571-5.

20. Lima FM, Hyde M, Chungkham HS, Correia C, Campos AS, Campos M, et al. Quality of life amongst older Brazilians: a cross-cultural validation of the CASP-19 into Brazilian-Portuguese. PLoS One 2014; 9:e94289.
21. Schneider IJC, Cofortin SC, Bernardo CO, Bolsoni CC, Antes D, Pereira KG, et al. Estudo de coorte EpiFloripaIdoso: métodos, aspectos operacionais e estratégias de seguimento. Rev Saúde Pública 2017; 51:104.

22. Marques LP, Schneider IJ, d'Orsi E. Quality of life and its association with work, the Internet, participation in groups and physical activity among the elderly from EpiFloripa survey, Florianópolis, Santa Catarina State, Brasil. Cad Saúde Pública 2016; 32:e00143615.

23. Tessari AA, Giehl MWC, Schneier IJC, Gonzales-Chica DA. Anthropometric measures change and quality of life in elderly people: a longitudinal population-based study in Southern Brazil. Qual Life Res 2016; 25:3057-66.

24. Lima-Costa MF, de Andrade FB, Souza PRB, Neri AL, Oliveira Duarte YA, Castro-Costa E, et al. The Brazilian Longitudinal Study of Aging (ELSI-BRAZIL): objectives and design. Am J Epidemiol 2018; 187:1345-53.

25. Beaton DE, Bombardier C, Guillemin F, Ferraz MB. Guidelines for the process of cross-cultural adaptation of self-report measures. Spine 2000; 25:3186-91.

26. Marôco J. Análise de equações estruturais. Fundamentos teóricos, software e aplicações. 2a Ed. Lisboa: ReportNumber; 2014.

27. Gouveia VV, Milfont T, Fonseca PN, Coelho JAPM. Life satisfaction in Brazil: testing he psychometric properties of the Satisfaction with Life Scale (SWLS) in five Brazilian samples. Soc Indic Res 2009; 90:267-77.

28. Damasio BF, Zanon C, Koller SH. Validation and psychometric properties of the Brazilian version of the Subjective Happiness Scale. Univ Psychol 2014; 13:17-24.

29. Ryff CD. Psychological well-being revisited: advances in the science and practice of eudaimonia. Psychother Psychosom 2014; 83:10-28.

30. Gale CR, Cooper C, Deary IJ, Aihie Sayer A. Psychological well-being and incident frailty in men and women: the English Longitudinal Study of Ageing. Psychol Med 2014; 44:697706.

31. Gu D, Feng Q, Sautter JM, Yang F, Ma L, Zhen Z. Concordance and discordance of self-rated and researcher-measured successful aging: subtypes and associated factors. J Gerontol B Psychol Sci Soc Sci 2017; 72:214-27.

32. Steptoe A, Deaton A, Stone AA. Psychological wellbeing, health and ageing. Lancet 2015; 385:640-8

33. Sexton E, King-Kallimanis BL, Layte R, Hickey A. CASP-19 special section: how does chronic disease status affect CASP quality of life at older ages? Examining the WHO ICF disability domains as mediators of this relationship. Aging Ment Health 2015; 19:622-33.

34. Liao J, Brunner EJ. Structural and functional measures of social relationships and quality of life among older adults: does chronic disease status matter? Qual Life Res 2016; 25:153-64. 


\section{Abstract}

The study aimed to investigate psychometric indicators of validity in the new version of CASP-19 for Brazilians 55 years and older and to study relations between scores on the scale and sex, age, schooling, and conjugal status. A total of 368 participants enrolled in educational programs for the elderly answered the CASP-19 scale (control, autonomy, self-realization, and pleasure), translated from English by five specialists and tested in 19 women $(\alpha=0.730)$. The data were submitted to exploratory factors analyses (EFA) and confirmatory factor analyses (CFA) using the structural equations method for latent variables and for internal consistency and correlation with scales of similar content. The scores were compared according to sex, age, schooling, and conjugal status. CFA produced a model with 19 items and 2 factors (self-realization/pleasure and control/autonomy), with good fit indices $(G F I=0.8 ; A G F I=0.7606$; $C F I=0.7241 ; N N F I=0.6876 ; S R M R=0.0902$; RMSEA = 0.0928; 90\%CI: 0.0827-0.1031). Cronbach's $\alpha$ was 0.837 for factor 1 and 0.670 for factor 2, and 0.874 on the total scale for individuals with 9 or more years of schooling, 0.834 for 5 to 8 years, and 0.772 for 1 to 4 years. High and significant correlations were observed with the scores in scales on satisfaction and subjective happiness. Men scored higher than women on selfrealization/pleasure; older individuals and those with the most schooling scored lower on control/ autonomy; individuals with a spouse or partner scored higher on control/autonomy. The new version of CASP-19 was effective in assessing self-rated quality of life in individuals 55 years or older in residents of the Southeast, South, and Northeast of Brazil.

Quality of Life; Reproducibility of Results; Aging

\section{Resumen}

ALos objetivos fueron investigar los indicadores psicométricos de validez en la nueva versión de la CASP-19 para brasileños con 55 años o más, así como estudiar relaciones entre las puntuaciones en la escala, sexo, edad, escolaridad y estatus conyugal. Trescientos sesenta y ocho usuarios habituales de programas educacionales para la $3^{a}$ edad respondieron a la CASP-19 (control, autonomía, autorrealización y placer), traducida y adaptada del inglés gracias a cinco especialistas, y probada en 19 mujeres $(\alpha=0,730)$. Los datos fueron sometidos a análisis factoriales exploratorios (AFE) y confirmatorios (AFC), mediante el método de ecuaciones estructurales para variables latentes; de consistencia interna y de correlación con instrumentos de contenido similar. Se compararon las puntuaciones de los grupos de sexo, edad, escolaridad y estatus conyugal. Los AFC produjeron un modelo con 19 items y 2 factores (autorrealización/placer y control/autonomía), con buenos indices de ajuste $(G F I=0,8 ; A G F I=0,7606$; $C F I=0,7241$; $N N F I=0,6876 ; S R M R=0,0902 ; R M S E A=$ 0,0928; IC90\%: 0,0827-0,1031. Los a de Cronbach fueron 0,837 para el factor 1 y 0,670 para el 2; 0,874 en la escala total para los de 9 años o más de escolaridad, 0,834 para los de 5 a 8 años y 0,772 para los de 1 a 4 años. Se observaron correlaciones altas y significativas con los marcadores en escalas de satisfacción y felicidad subjetiva. Los hombres tuvieron una puntuación más alta en autorrealización/placer que las mujeres; los más viejos y más escolarizados, una puntuación más baja en control/autonomía; los sin cónyuge, más alta en control/autonomía. La nueva versión de la CASP-19 fue eficaz para evaluar la calidad de vida percibida en individuos de 55 años o más, residentes en las regiones sudeste, sur y nordeste del país.

Calidad de Vida; Reproducibilidad de los

Resultados; Envejecimento
Recebido em 16/Out/2017

Versão final reapresentada em 19/Mar/2017

Aprovado em 18/Mai/2018 